\title{
Neuro-Behcet disease
}

Lin Zhang ${ }^{1,2}$, Xiaopeng Wang ${ }^{1}$, Fan Liu' ${ }^{1}$, Zhuo Zhang ${ }^{1}$

${ }^{1}$ Department of Neurology, The Second Hospital of Hebei Medical University, Rizhao Central Hospital, Shijiazhuang, China

2Department of Neurology, Rizhao Central Hospital, Shijiazhuang, China

Submitted: 11 December 2020; Accepted: 16 January 2021;

Online publication: 26 February 2021

Arch Med Sci 2021; 17 (2): 569-573

DOI: https://doi.org/10.5114/aoms/132471

Copyright $\odot 2021$ Termedia \& Banach

Behcet's disease (BD) is commonly seen in East Asia, the Middle East and the Mediterranean, known as "Silk Road disease". It is more common in our country, mostly involving young people. The incidence rate of stroke in young people is increasing gradually, and the cause is complicated. Vasculitis caused by neuro-Behcet's disease (NBD) plays an important role. Many researchers have reported many cases of NBD, and analyzed the relationship between stroke and Behcet's disease. However, the diagnostic criteria of NBD were not unified, the clinical manifestations were diverse, and imaging and laboratory examinations were not specific. The incidence rate of different areas in clinical practice varied greatly, and the misdiagnosis rate was high. Therefore, attention should be paid to the early diagnosis and active treatment of NBD.

This article presents a case of NBD with definite diagnosis. We aimed to explore the clinical manifestations, laboratory tests and differential diagnosis of NBD through this case.

This study was conducted with approval from the Ethics Committee of the second hospital of Hebei Medical University.

Case 1: A 33-year-old man was admitted to hospital on August 21, 2015, suffering from paroxysmal dizziness and walking to the left for $40 \mathrm{~h}$. The patient had no obvious prior cause of the dizziness. Other symptoms included distension of the head, which lasted for about $30 \mathrm{~min}$, and which was then completely relieved but followed by further intermittent attacks. CT showed no obvious abnormality, but MRI showed abnormal signal of the left cerebellar hemisphere, demonstrating acute cerebral infarction (Figure $1 \mathrm{~A}$ ). Concerning inflammation of the left maxillary sinus: The left anterior inferior cerebellar artery was thinner than the contralateral side, and the bilateral posterior inferior cerebellar artery was not developed. MRA showed that other intracranial arteries were normal (Figure $1 \mathrm{~B}$ ).

The patient had a previous history of herpes zoster neuralgia, oral tumor resection, and duodenal ulcer.

Physical examination: T3 $6.6^{\circ} \mathrm{C}, \mathrm{P} 74$ beats $/ \mathrm{min}, \mathrm{R} 18$ times/min, BP $118 / 73 \mathrm{~mm} \mathrm{Hg}(1 \mathrm{~mm} \mathrm{Hg}=0.133 \mathrm{kPa})$. Physical examination of the heart, lung and abdomen showed no abnormalities. The abdomen was soft, without tenderness or muscle tension. There was no edema in both lower limbs and no history or family history of infection.

Physical examination of nervous system: clear thoughts, good language, normal reaction and orientation. The pupil was large and had an equal circle with a diameter of about $3.0 \mathrm{~mm}$. The pupil was sensitive to light reflection. The eyeball moved freely in all directions without nys-

\author{
Corresponding author: \\ Xiaopeng Wang \\ Department of Neurology \\ The Second Hospital of Hebei \\ Medical University \\ Shijiazhuang 050000, China \\ Phone: 15803213931 \\ E-mail: zhanglindrr@163.com
}



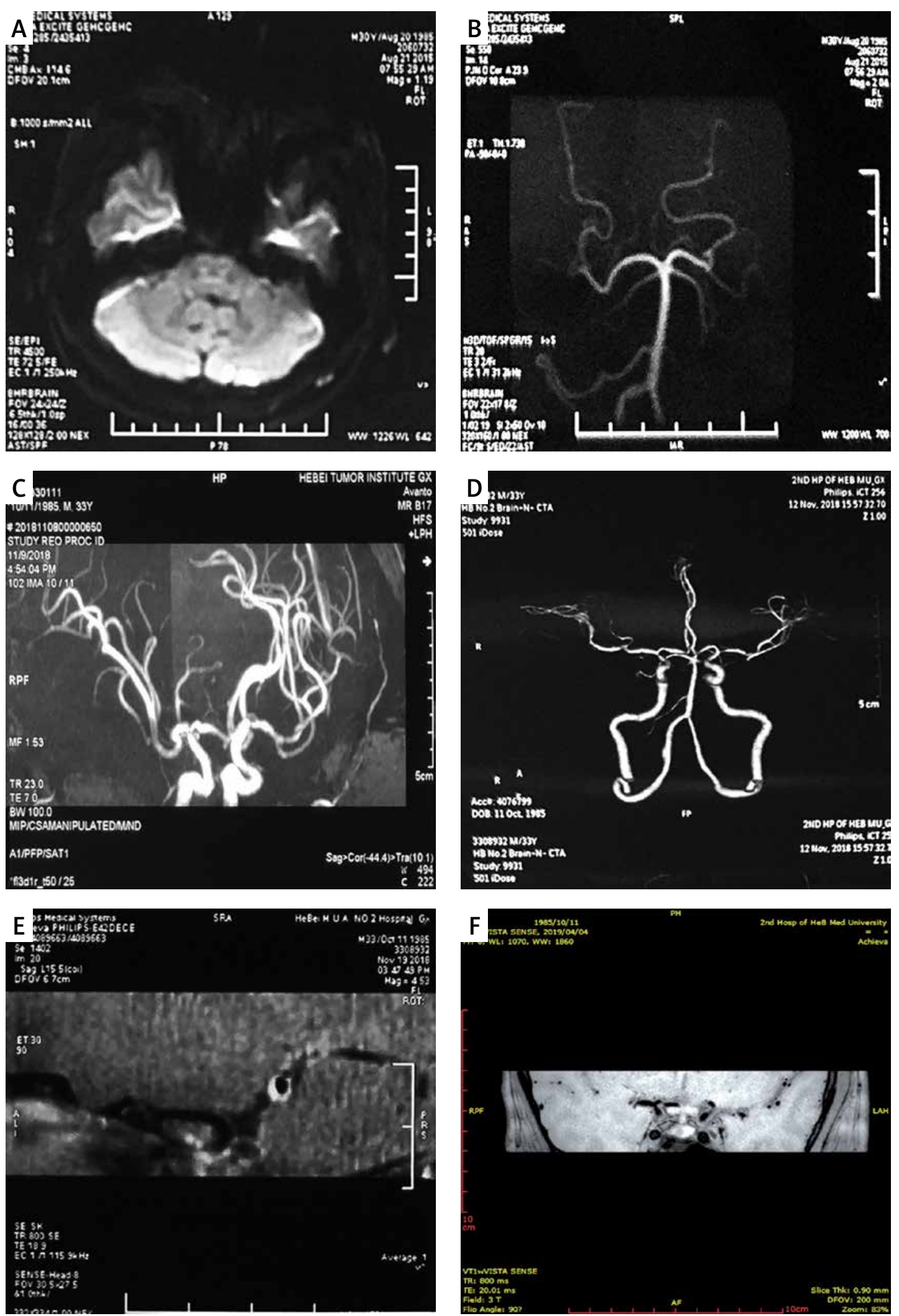

Figure 1. A - August 21, 2015. DWI examination of left cerebellar acute cerebral infarction in our hospital. B - PostMRA cycle in our hospital on August 21, 2015. C - MRA arteriosclerosis and multiple stenosis in the fourth hospital on November 9, 2018. D - Right vertebral aneurysm of CTA in our hospital on November 12, 2018. E - Inflammatory changes in bilateral middle cerebral arteries of HR-MR in our hospital on November 19, 2018. F - Inflammatory changes in bilateral middle cerebral arteries of HR-MR in our hospital on April 4, 2019 

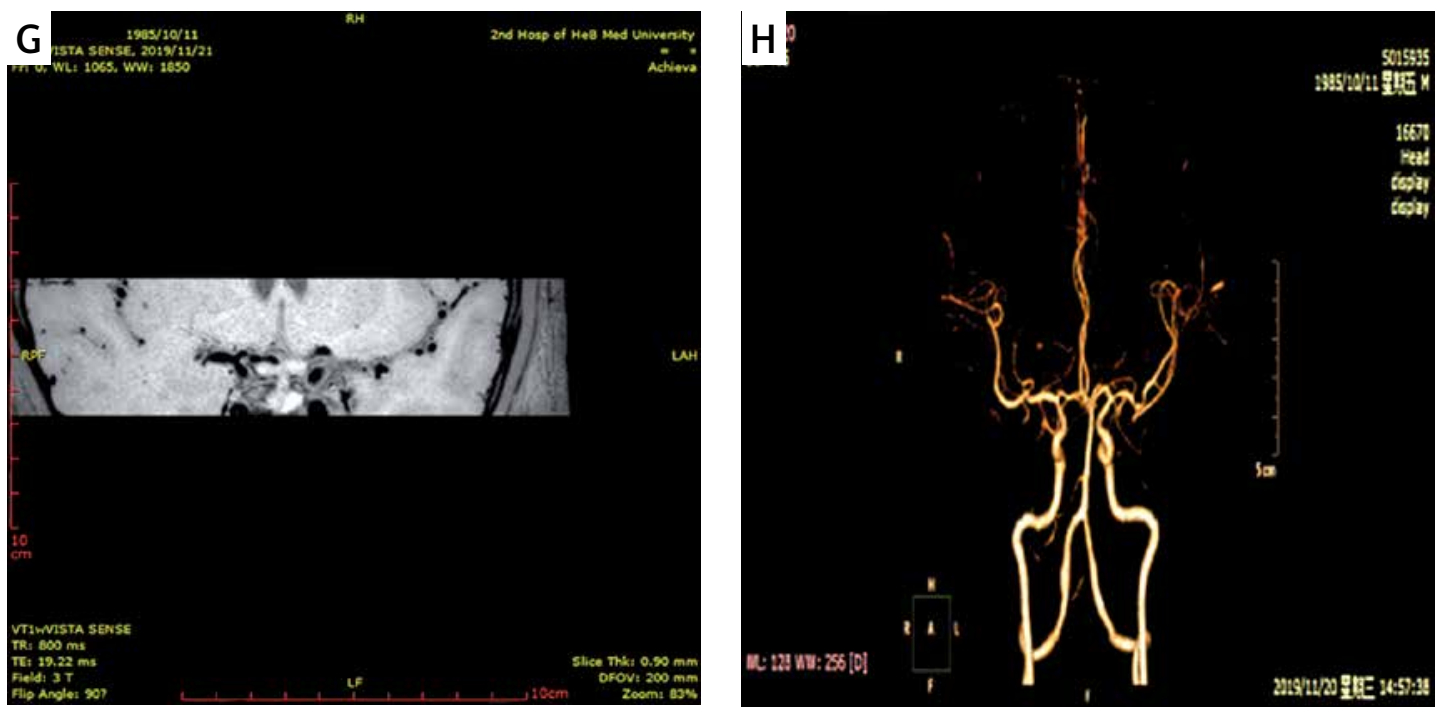

Figure 1. Cont. G - Inflammatory changes in bilateral middle cerebral arteries of HR-MR in our hospital on November 19, 2019. H - On November 20, 2019, CTA in our hospital showed a narrowing of the lumen of the left middle cerebral artery

tagmus. Bilateral frontal lines were symmetrical, bilateral nasolabial sulci were symmetrical, and tongue extension was in the middle. The muscle strength of the limbs was grade $\mathrm{V}$, and the muscle tension of the limbs was normal. Limb tendon reflex $(++)$, bilateral pasteurization $(-)$. No obvious abnormality or ataxia was found in the sensory system. Neck resistance (-). NIHSS score: 0. Swallowing function: Grade 1.

Laboratory tests: Homocysteine $16.4 \mu \mathrm{mol} / \mathrm{l}$ (normal value 0-15 $\mu \mathrm{mol} / \mathrm{l}$ ), lipoprotein phospholipase A2 $338.0 \mathrm{ng} / \mathrm{ml}$ (normal value: 0-175 ng/ml).

Neck vascular ultrasound: Right subclavian artery local intima-media thickening.

Diagnosis: Cerebellar infarction. Treatment after hospitalization: Smoking cessation, low salt and low-fat diet, oral clopidogrel $75 \mathrm{mg} /$ day, atorvastatin $20 \mathrm{mg} /$ day, butylphthalide injection $25 \mathrm{mg} /$ day, calf serum injection $1.2 \mathrm{~g} /$ day, oxiracetam $4.0 \mathrm{~g} /$ day. The symptoms of dizziness improved at discharge.

Case 2: The patient took clopidogrel, atorvastatin, and butylphthalide regularly outside the hospital and stopped taking drugs 1 year later without any discomfort. On November 6, 2018, the patient suffered dizziness again, and the EEG showed extensive and mild abnormalities. Brain MR showed that the head MRI was consistent with arteriosclerosis and had multiple vascular localized stenosis (Figure $1 \mathrm{C}$ ) and left maxillary sinusitis.

Physical examination showed no positive signs.

Laboratory tests: Lam antibody of mycobacterium tuberculosis was weakly positive, IFN- $\gamma$ was positive, citric acid bacteria Klebsiella was cultured in sputum, PPD test was $(++)$.

Vascular ultrasound: Right subclavian artery, bilateral common femoral artery, left popliteal artery plaque formation, left axillary artery, brachial artery, radial artery and ulnar artery wall was not smooth.

CT head and carotid artery: The intracranial segment of the right vertebral artery was aneurysmally dilated, suspected aneurysm, and multiple local lumens of M1-2 segment of left middle cerebral artery were narrowed (Figure $1 \mathrm{D}$ ).

High resolution magnetic resonance imaging (HR-MRI): The wall of the M1 segment of the bilateral middle cerebral artery was not evenly thickened, with uneven enhancement, and the corresponding left proximal segment lumen was slightly narrowed, and no obvious stenosis was found in the other corresponding lumens, which was consistent with inflammatory changes of the bilateral middle cerebral arteries (Figure $1 \mathrm{E}$ ).

Dynamic contrast enhanced magnetic resonance imaging (DCE-MRI): There was no significant difference in Ktrans value or VP value in the bilateral middle cerebral artery blood supply area, indicating that the blood-brain barrier was not significantly damaged.

Chest CT: Left upper lobe apex posterior segment lesions, excluding tuberculosis; bilateral pleural thickening, a little effusion in the right chest.

Diagnosis: Intracranial vasculitis? Old cerebral infarction, intracranial aneurysm, secondary pulmonary tuberculosis. Post-hospital treatment: Low salt and low-fat diet, oral aspirin 100 mg/day, atorvastatin $20 \mathrm{mg} /$ day, butylphthalide injection $25 \mathrm{mg} /$ day, isoniazid, rifampicin, pyrazinamide, ethambutol, silybin meglumine and other drugs. The symptoms of dizziness improved at discharge.

Case 3: The patient was diagnosed with Behcet's disease at the Department of Immunology and Rheumatology at the Peking Union Medical College Hospital. Prednisone acetate 50 mg/day, 
azathioprine 200 mg/day, calcitriol 0.25 g 1/day, isoniazid $300 \mathrm{mg} /$ day were given orally. Prednisone acetate was gradually reduced to $5 \mathrm{mg} \mathrm{1/day}$.

On April 4, 2019, high resolution MR showed inflammatory changes in bilateral middle cerebral arteries (no official report) (Figure $1 \mathrm{~F}$ ).

Lower extremity artery ultrasound: Bilateral common femoral artery, left popliteal artery plaque formation.

Family history: Patient's sister had a history of similar ulcers.

Laboratory test: RVVT normalized ratio of lupus anticoagulant was 1.17 (normal value: 0.93-1.11).

Vascular ultrasound: Left femoral vein valve insufficiency, right common femoral artery, bilateral popliteal artery, bilateral dorsal pedal artery plaque formation, left common femoral artery local intima-media thickening.

High resolution MRI: Considering the atherosclerotic inflammatory changes of the $\mathrm{C} 7$ segment of the right internal carotid artery and the origin of the middle cerebral artery and M1 segment of the left middle cerebral artery, some branches of the $M 2$ segment of the bilateral middle cerebral artery and the small perforating artery underwent enhanced scanning with circular enhancement (Figure $1 \mathrm{G}$ ).

CT head and carotid artery: The intracranial segment of the right vertebral artery was aneurysmally dilated, with suspected aneurysm, and the lumen of the M1-2 segment of the left middle cerebral artery was narrowed (Figure $1 \mathrm{H}$ ).

Chest CT: Local lesions in the apical segment of the left upper lobe were considered, and a few hypostatic changes were found in the back of both lungs.

Oral administration of prednisone acetate, azathioprine, calcitriol, isoniazid, vitamin D calcium, atorvastatin and other treatments. The symptoms still recurred from time to time.

Behcet's disease is a chronic disease with unknown etiology that can involve multiple systems. It is characterized by oral ulcer, vulvar ulcer, ophthalmia and skin damage. Its basic pathological change is vasculitis. According to the varying damage to the visceral system, BD can be divided into vascular type, nerve type, gastrointestinal type, etc. Nerve type refers to those types with central or peripheral nerve involvement, especially the central nervous system, with only limited peripheral nervous system involvement [1]. The most common clinical manifestation of NBD is headache [2] It can be divided into the parenchymal type and non-parenchymal types [3]. The parenchymal type mainly involves the brainstem, spinal cord, and cerebral hemisphere. The non-parenchymal type is also called vascular NBD, which includes venous sinus thrombosis and aneurysm [1].

The common pathological changes of NBD are as follows: vasculitis can be seen in the affected area, and a large amount of lymph and monocyte infiltration can be seen around the blood vessels. Exudative lesions are manifested as lumen congestion, wall edema, endothelial cell swelling, and fibrin deposition, leading to the disappearance of local small blood vessels and fibroid degeneration. Proliferation of endothelial cells and adventitia cells, thickening of the tube wall, and necrotizing granuloma could be seen in severe cases. The deposition of immunoglobulin and complement could be seen under the immunofluorescence microscope.

The clinical manifestations of NBD are mainly meningoencephalitis, fever, meningeal irritation, and the corresponding symptoms of the cerebral cortex, brainstem and cerebellum damage, benign intracranial hypertension, spinal cord damage, and peripheral neuropathy [4]. Recurrent oral ulcer is the first and essential symptom of BD, which can attack more than 3 times per year and could been seen in $98 \%$ of NBD patients [5]. Other skin and mucosa lesions including erythema nodosum, pseudofolliculitis, acne-like folliculitis, superficial thrombophlebitis, erythema multiforme, erythema annularis, and necrotizing tuberculous eruption could also been seen in NBD patients. Ophthalmitis, such as uveitis, retinitis, conjunctivitis, corneal ulcer, choroiditis, and optic neuritis, can cause serious visual impairment and even blindness of NBD patients. NBD can also affect the digestive system, cardiovascular system, joint system, and respiratory system, causing diseases such as peptic ulcer, asymmetric arthritis, and tuberculosis infection [6].

The non-steroidal anti-inflammatory drugs colchicine and glucocorticoid can be used for symptomatic treatment in BD patients. Vasculitis of the visceral system is mainly treated with glucocorticoid and immunosuppressants. CTX, MTX, AZA and cyclosporine are commonly used [7]. Biological agents may be an effective alternative therapy for NBD. Non-parenchymal NBD, such as venous sinus thrombosis or isolated intracranial hypertension, should be treated with anticoagulation and intracranial pressure reduction. However, hormones and immunosuppressants can cause adverse reactions. If they are taken for an extended period, the dosage must be adjusted according to the clinical manifestations and the blood tests. Liver and kidney function, blood glucose, and blood pressure should be closely monitored. In the case of abnormality, reduce the dosage, stop the drug, or use alternative drugs. Most patients had a good prognosis [2].

The differential diagnosis of NBD includes primary Sjogren's syndrome, multiple sclerosis, Fabry's disease and Moyamoya disease. For primary Sjögren's syndrome, fever and joint pain may appear, but most patients have typical symptoms of dry eye and mouth, which can be differentiated 
by labial gland biopsy [8]. Multiple sclerosis is an autoimmune disease characterized by inflammatory demyelinating lesions in the central nervous system. Its main clinical features are multiple foci scattered in the central nervous system white matter and remission and recurrence in the course of the disease, multiple spatial and temporal symptoms and signs, which can be distinguished from abnormalities of the oligoclonal zone, AQP4, MOG and MBP in cerebrospinal fluid [9]. $37 \%$ of patients with Fabry's disease have symptoms of central nervous system damage, the onset age is generally more than 26 years old, and there is occurrence of stroke (24\%), dementia, passive and depressive social activity disorder and other personality changes (18\%). Cerebrospinal fluid examination is normal, and brain magnetic resonance imaging (MRI) examination can detect small lesions in white matter and gray matter early [10]. Moyamoya disease is a kind of cerebrovascular disease with unknown etiology, characterized by chronic progressive stenosis or occlusion at the ends of bilateral internal carotid arteries, anterior and middle cerebral arteries, and secondary formation of an abnormal vascular network in the skull base [11]. It is another cause of youth stroke, leading to persistent paralysis, aphasia, visual impairment and mental retardation.

In conclusion, the patient was a young man with a long course of disease, presenting with recurrent dizziness. He was diagnosed with $\mathrm{BD}$ at the Peking Union Medical College hospital, and received hormone and immunosuppressant treatments. The patient's symptoms improved significantly. For the third time, the symptoms of dizziness worsened and recurred. The laboratory test in the Department of Immunology and Rheumatology of our hospital showed that the standardized ratio of RVVT of lupus anticoagulant increased. Also the intracranial and extracranial vascular imaging examination showed that the vascular condition was worse than before. The patient's medical history, duodenal ulcer, recurrent oral ulcer (mainly in summer) and back folliculitis were inquired about. To sum up, the patient was diagnosed with NBD with sufficient evidence. However, the patient has some special features. Oral ulcer and back folliculitis recur in summer, and the patient is hospitalized with dizziness in early winter every time. During the hospitalization, there is no ulcer and folliculitis, and the skin manifestation of BD is separated from the symptoms of cerebrovascular disease.

Behcet's disease is an immune disease that can involve multiple systems. Is BD related to a previous history of herpes zoster neuralgia, oral tumor resection, duodenal ulcer, pulmonary tuberculosis, and emotional susceptibility? Is the seasonal separation of BD skin manifestations and cerebrovas- cular disease symptoms special? For some young stroke and other neurological diseases, we should consider whether it is related to immune diseases. We hope that innovative detection methods will assist in future diagnoses.

\section{Acknowledgments}

Lin Zhang and Xiaopeng Wang contributed equally to this work.

\section{Conflict of interest}

The authors declare no conflict of interest.

\section{References}

1. Lin CH. The clinical progress of neuro-Behcet's disease. Fudan University Journal of Medical Sciences 2018; 45: 260156-60.

2. Qian XQ, Shi XQ. Diagnosis and treatment of neuro-Behcet's Disease. Med Recapit 2017; 23: 3837-42.

3. Yang QL, Jiawei W, Chuntao L. Stroke related to Behcet's disease. Chin J Stroke 2017; 12: 57-60.

4. Noel N, Bernard R, Wechsler B, et al. Long-term outcome of neuro-Betch's disease. Arthtitis Rheumatol 2014; 66: 1306-14.

5. Shi YY. Clinical analysis of 32 cases of neurobehcet's disease. J Changzhi Med Coll 2017; 31: 271-3.

6. Qi J, Liu J, Wu YG, Zhou XH. A case report of Behcet's disease with a history of Mycobacterium tuberculosis. Chinese J Neuropsych Dis 2017; 43: 52-3.

7. Joseph FG, Scolding NJ. Neuro-Behcet's disease in Caucasians: a study of 22 patients. Eur J Neurol 2007; 14: 174-80.

8. Marshall LL, Stevens GA. Management of primary Sjögren's syndrome. Consult Pharm 2018; 33: 691-701.

9. Oh J, Vidal-Jordana A, Montalban X. Multiple sclerosis: clinical aspects. Curr Opin Neurol 2018; 31: 752-9.

10. Zarate YA, Hopkin RJ. Fabry's disease. Lancet 2008; 372: 1427-35.

11. Hishikawa T, Sugiu K, Date I. Moyamoya disease: a review of clinical research. Acta Med Okayama 2016; 70: 229-36. 